\title{
Considerations Regarding the Analysis and Interpretation of Traumatic Lesional Mechanisms in Road Accidents
}

\author{
Lucian Tarnu ${ }^{1, *}$, and Cristian Deac ${ }^{1}$ \\ ${ }^{1}$ Lucian Blaga University of Sibiu, Faculty of Engineering, E. Cioran str. 4, 550025 Sibiu, Romania
}

\begin{abstract}
According to statistics, road transportation is the most dangerous means of transportation and also the costliest in terms of human lives and material damage. The increase of road safety is a priority goal of the European Commission and an area of high interest for Romania, which is unfortunately placed very high in the hierarchy of countries with a high number of deaths resulting from road accidents. This study presents the mechanisms of the generation of traumatic injuries and also analyses the manner of interpreting these mechanisms in the case of road accidents, both from the perspective of law enforcement and from the perspective of specialists in road transportation, who analyse and investigate road accidents from a technical point of view. Determining the lesional mechanisms helps to better understand the real image of the conditions of an accident's occurrence, in order to clearly illustrate the elements mentioned in the forensic accident site report and to complement all other means to gather information at that site.
\end{abstract}

\section{Introduction}

The increase in the volume of road traffic worldwide, has led also to road safety becoming a major problem that has to be addressed in every country. Currently, the recommended method for tackling this problem is prevention, which requires a thorough assessment of the impact of various factors, starting from the various categories of human participants in the road traffic: car drivers, pedestrians, bicycle riders etc. and the vehicles themselves - trucks, cars, motorcycles, bicycles etc., but including also the elements of transportation infrastructure (roads, bridges, road signals etc.) and not least the different types of road accidents with their causes, consequences, but also the manner of their unfolding.

Road accidents are a major source of social and economic problems and therefore need to to be recorded and analysed in all their aspects so as to better understand them, but also work towards preventing future similar accidents and/or minimizing the consequences of accidents if they happen. In many cases, road accidents impact the jobs, health or even life of those involved, so road accidents can be viewed as work accidents and risks related to road safety can be assessed using a methodology similar to that used for determining the risks at the workplace [1].

* Corresponding author: cristian.deac@ulbsibiu.ro 
Innovations in areas such as renewable fuel sources [2, 3], safer driving systems or road infrastructure can help to reduce the risk of road accidents, but it is also important for all participants in road traffic to understand the manner in which road accidents occur and what their consequences are.

The European Commission has set, first in 2001 and then in 2011, ambitious goals for reducing in 10 years the number of road fatalities by 50\%. In 2017 there had been recorded a reduction of the number of fatalities in road accidents by $20 \%$ compared to 2011 (from 31500 to 25260$)[4,5,6]$.

While similar studies have been carried out from a statistical, theoretical point of view, e.g. $[7,8,9]$, or with focus only on specific injuries, e.g. head injuries in [10], in this paper, the authors present, both from a theoretical and practical point of view, the mechanisms and manners by which traumatic injuries can occur during severe road accidents. These can be important elements since, in some cases, the traumatic injuries determined through medical legal examination, even of masked as a traumatism caused by road accidents, can suggest other ways and circumstances for the event's occurence.

\section{Lesional Mechanisms}

The genesis of traumatic injuries noticed on victims of road accidents, regardless of their role - e.g. car driver, pedestrian, bicycle rider, passenger etc. can be explained through three categories of mechanisms: simple mechanisms, associated mechanisms and complex mechanisms.

Simple mechanisms, generated by a single action on the human body, are only rarely encountered, while the associated mechanisms and especially the complex mechanisms are much more frequent.

While associated mechanisms determine traumatic injuries that can be interpreted by combining two simple mechanisms, the complex mechanisms imply the combination of at least three simple mechanisms that can explain the encountered lesional polymorphism.

\subsection{Simple Mechanisms}

\subsubsection{Hitting (Impacting)}

This lesional mechanism is the most frequent, since in most cases a part of the vehicle come sinto direct contact with the victim's body, actively producing the traumatic injuries at the impact site.

In such situations, the traumatic injuries are unipolar, being more frequently encountered on the lateral parts or on the back side of the human body, where the impact had occured and have a reduced severity.

Most often there are noticed elementary traumatic injuries, such as bruises, abrasions, wounds and osteo-articular injuries such as sprains and fractures. When fractures occur, they affect especially the pelvian members, in the case of pedestrians, or the head-thorax assembly for persons inside vehicles.

In the particular case of the active hitting of a person, either as pedestrian, or as passenger/driver in a vehicle, with a hard object such as a stone that might be ejected from the road by the wheel of another vehicle, the traumatic injuries will be localised in the impact area and will usually affect the upper part of the body, with various degrees of severity function of the impact force [9]

If the victim wears thick clothing, the vehicle's speed is reduced and the area of impact with the vehicle is relatively large, the traumatic injuries may even be absent. Generally, for 
the same speed, the impact with larger areas generates less severe injuries than impacts with smaller areas, for which more severe injuries are reported.

When the impact occurs below the human body's centre of gravity, usually there can be noticed a post-impact rotation of the body, leading to traumatic injuries occurring also on the opposed lateral part of the body, e.g. by hitting parts of the car chassis or by hitting the ground.

When the impact occurs above the human body's centre of gravity, such as in the case when large-size vehicles are involved, there can be noticed a translation motion of the body, followed by falling or throwing.

\subsubsection{Falling}

This lesional mechanism is encountered almost exclusively in the case of drivers or passengers in vehicles, either as a consequence of accelerating or as a consequence of a sudden stop (deceleration) and explains the traumatic injuries after a passive impact between the body and a hard plane on which the victim falls.

Function of the particularities of the plane on which the fall occurs: horizontal or sloped, smooth or rough, of the distance to the plane and the vehicle's speed, the injuries may be more or less severe, but generally unipolar, while the elementary injuries are quasiconstant.

In such situations, the incidence of fractures is much higher, while the internal organs are affected only sporadically.

The hypothetical situation of a pedestrian falling, either because of the drag generated by a vehicle passing by at high speed or because the pedestrian panicked and wishing to avoid the vehicle, tripped and fell, while possible, is much rarer in practice. This type of situations implies a direct correlation of the factors involved in the juridical solving of the case, taking into account also a legal medical opinion. [9]

\subsubsection{Projecting}

This mechanism is the most frequent both in pedestrians and in the drivers/passengers of vehicles. While in the case of pedestrians, projecting is preceded by an impact, in the case of persons in vehicles, projecting occurs without initial impact.

Persons in vehicles may be projected inside of vehicles, as is frequently the case of sudden decelerations, hitting the interior parts of the vehicle or the other persons within the vehicle (in cars, the passenger in the back may be projected into the head of the person in front of him, leading to traumatic cranial-cerebral injuries for both) or outside the vehicle, such as in the case of a truck entering a curve with a too high speed and leading to the passengers on the truck's platform being projected onto the roadside. Projecting may lead to both external and internal traumatic injuries.

While the external traumatic injuries, although being spread on a larger area, multiple, diverse and multipolar are less severe, the internal traumatic injuries are more severe, consisting of fractures, visceral ruptures and hemmorrhage and being mostly lethal.

In the case of pedestrians, the initial injuries, produced by impact, will affect especially the lower part of the body and have to be searched for carefully and individualised, especially since it might be included in a lesional polymorphism induced by the projecting mechanism. Only by identifying this traumatic first contact injury can the traumatism directly resulting from the road accident (impacting) be separated from the traumatism caused by projecting, which from a legal point of view may involved distinctive consequences. [9] 


\subsubsection{Compressing}

This lesional mechanism, implying the body's crushing between one side of the vehicle and another hard surface, such as a wall, a tree, the ground (road) or another vehicle. Frequently, when compression occurs between a vehicle and the ground, the dragging mechanism is also present.

Function of the compression's intensity, the victim's body will present, beneath oil marks, paint marks or other types of marks from the vehicle, also traumatic injuries characterised by bipolarity as as consequence of the two opposed hard planes between which the body is compressed.

Elementary injuries are reduced, with precise localisation and represented especially by bruises and abrasions generated by the metallic profiles of the vehicle or by the active compression plane and/or by the roughness of the passive contact plane.

On the other hand, internal traumatic injuries are severe, often being encountered bilateral rib fractures and double basin fractures. In the case of severe compression, visceral ruptures may occur, followed by internal bleeding.

For compression, there has to exist a lesional correspondence between external and internal traumatic injuries; in its absence, the compression mechanism is uncertain.

\subsubsection{Dragging}

This mechanism consists in the victim being caught by a vehicle part and then dragged over a hard surface. In practice, dragging is rarely encountered on its own, being usually a part of associated or complex mechanisms. Regardless of whether they were initially within a vehicle or whether they were pedestrians, the victims will present primarily external traumatic injuries. These will be more or less visible, function of the distance and of the roughness of the plane on which the dragging occured, also function of the victims' clothing and of the vehicle's speed. Injuries are usually unipolar, represented by:

- parallel band-like abrasions, generated by the skin's friction on the plane on which the dragging occurs; they are more visible on the side opposed to the area on which the body was caught on the vehicle and have a reverse direction to the vehicle's movement direction;

- superficial small-size wounds, occurring as irregular striations, perpendicular to the dragging direction, explainable through the stretching and later bursting of the skin.

In the case in which the vehicle's movement direction is not linear and the dragging plane is rough, beneath the extensive abrasive injuries that imitate the aspect of traumatic injuries after burns (from which they can be differentiated by the fact that in burns, the hairs are modified), there may occur also internal injuries that, when affecting the head, may lead to death.

\subsubsection{Running over}

This mechanism is associated with the movement system of the vehicle (wheel, track, gliding sole), over the victim's body, thus being different from the compressing mechanism that implies, as mentioned, the body's crushing between one side of the vehicle (different from the movement system) and another hard plane.

In most cases, traumatic injuries produced by running over are quickly lethal through hemorrhage after the sectioning of important blood vessels or through the crushing of vital organs. Usually, the skin retains its integrity, even when it was run over by a metal wheel, due to the tegument's elasticity. In the case of bodies, the area becomes pergament-like. 
More frequently than in other simple mechanisms, in the case of running over, the traumatic injuries produced to the victims while they were alive have to be differentiated from those produced post-mortem. The vital character of traumatic injuries thus becomes essential in differentiating for example between a homicide with the subsequent placement of the body on a road or a railway track, and the situation when a pedestrian who was hit by a vehicle and fell unconscious onto the road, where they were abandoned by one driver who fled the accident site, is being run over and killed by another vehicle. In most cases, through a detailed post-mortem legal medical analysis, the vital reaction can be determined (quantitatively and qualitatively) so as to allow informed assumptions on the succession of the traumatic injuries' generation and thus on the conditions in which the death occured.

A forensic and/or legal medical examination can determine elements specific for each type of movement system.

a) Running over with a rubber tire

This can generate, as characteristic elements:

- Zigzag bruises, stripes in the movement direction of the wheel and reproducing the grooves on the tire's surface; these traumatic injuries, occurring when the compression force is so big that it leads to the body's crushing, are explained through the pushing of the soft tissues and the subsequent rupture of blood vessels in the grooves that form the tire's profile;

- Imprinting of the tire's profile, through the same mechanism as described above, when the tire passes tangentially; in these situations there can be usually noticed abrasions;

- Tegument ruptures and subsequent pocket-type or even scalped wounds when the tire initially slides before the actual running over occurs;

- Wide abrasion areas, that in dead bodies appear as pergament-like, explainable through the tire's friction on the skin prior to its running over the body.

b) Running over with a metal wheel (train, tram etc.)

In this case, the particularities of traumatic injuries are due to the compression between the two hard planes (wheel and metal rail). The most frequent traumatic injuries occurring are:

- The abrasion area (strip), generated either by the rolling track of the wheel (larger, approximately $12-15 \mathrm{~cm}$ ), or by the flange (smaller, approximately $2-3 \mathrm{~cm}$ ), occurs through a crushing-friction action; when the sectioning of that body part also occurs: the abrasion area is located along the section's border and has a width of a few centimetres;

- The strifing area (strip), placed on either side of the abrasion area, is produced by the friction between the wheel's (inner and outer) side surfaces and the skin; this area, with irregular margins, is dirty with oil and, from place to place, it displays abrasions or superficial wounds in the shape of circle arcs, imprinted by the profile on the side surfaces of the wheel (screws etc.);

- "The primary pinching " - T or "!"-shaped abrasion that can be sometimes noticed at the beginning of the abrasion area; it is explained through the initial compression action of the rolling track and the flange; in some cases, instead of the "primary pinching" there can be noticed two parallel abrasions, separated by an area of intact skin and generated by the rails's end;

- the cuneiform shape - lack of substance with the base towards the wheel and the tip towards the rail, occurring within the abrasion area; when the body or the member is not completely sectioned, tissular bridges formed of compressed skin and soft tissues will persist; 
- triangular skin flaps, determined by the rolling track or by the wheel's flange, with the tip towards the rolling direction, located at the sectioning border, only on the body area exposed to the wheel.

c) Running over with a metal track

The track is shaped like a closed chain within which the links are metal plates joined by metal rods; on the outer surface (which provides the movement on the ground), the track has bumps whose size, shape and distancing are characteristic for each type of track. The traumatic injuries are represented by:

- wounds, abrasions or (rarely) bruises, showing up as discontinuous strips, perpendicular on the rolling direction and parallel to each other, caused by the bumps; the wounds, with more or less regular borders, function of the bumps' sharpness, present traces of soil, grass etc., that were caught in the vehicle's movement;

- uneven tissue crushings, interrupted by deeper ruptures produced by the bumps, with traces of soil, grass or grease originating on the track;

- $\quad$ step fractures of the long bones, when the track (through the bumps) moves along that member segment.

d) Running over with the gliding sole of a sled

Most often, soles are made of wood or metal and, due to the relatively low weight of these transportation means, the traumatic injuries produced by them are mostly not lethal. As a characteristic element, there is an abrasion strip with a width equal to that of the gliding sole, on the movement direction of the sled and determined by its friction with the tegument; rarely, the abrasion strip is replaced by a bruised strip.

\subsubsection{Hyperextension-hyperflexion of the spine}

This lesional mechanism is frequently involved in producing vertebral-medular traumatic injuries, especially in passengers/drivers in cars, as as consequence of the sudden deceleration (braking) or acceleration of the vehicle. In such situations, there can occur the telescoping of the spine, with a major lesional resonance at the level of the spinal marrow.

\subsection{Associated Mechanisms}

As resulting from their name, in the genesis of traumatic injuries, for associated mechanisms two simple mechanisms are grouped. Usually, a legal medical examination will separate each mechanism.

The most frequent associated mechanisms are:

- impacting-falling

- impacting-compressing

- $\quad$ impacting-projecting

- proiecting-running over

- projecting-compression

- projecting-dragging

- dragging-compression

- running over-dragging etc. 


\subsection{Complex Mechanisms}

Complex mechanisms combine three or more simple mechanisms.

Traumatic injuries produced by complex mechanisms are characterised by:

- $\quad$ high severity, in most cases the victims dying;

- presence of osteo-articular injuries;

- multipolarity.

The distribution of traumatic injuries on several planes makes the individualisation of each simple mechanism impossible.

Among the most frequent complex mechanisms there can be mentioned:

- impact-projecting-running over;

- impacting-projecting-compressing and/or running over;

- projecting-compressing/running over-dragging;

- projecting-tilting-falling/compressing etc.

\section{Importance of determining the lesional mechanisms after road accidents}

In the following the authors present three actual cases whose analysis, carried out with the involvement of the authors and especially the correct identification of the lesional mechanisms involved, enabled reaching the correct conclusions with regard to the manner of the accident's occurrence and with regard to the party presumed to be guilty.

\subsection{First case study: impacting-falling-dragging complex mechanism}

The first case is a fatal accident that happened on January $22^{\text {nd }}, 2021$ on the A1 SibiuArad highway, not far from the exit from the city of Sibiu, Sibiu county and was ruled initially to be a road accident with unknown author. Identifying the lesional mechanism was very important for determining the conditions of the event and then for the identification of the vehicle involved in the accident.

In order to determine the lesional mechanism, there was carried out a forensic examination of the victim's injuries and of the victim's clothes.

The forensic examination of the victim's clothes revealed traces of oil and soil, also noticing the presence of several rips, as follows:

- the victim's trousers (Figure 1) presented traces of a black oily substance and of soil, as well as material ripping.

- the victim's jacket (Figure 2) presented traces of a brown-reddish blood-type substance, as well as ripping and separation of the material.

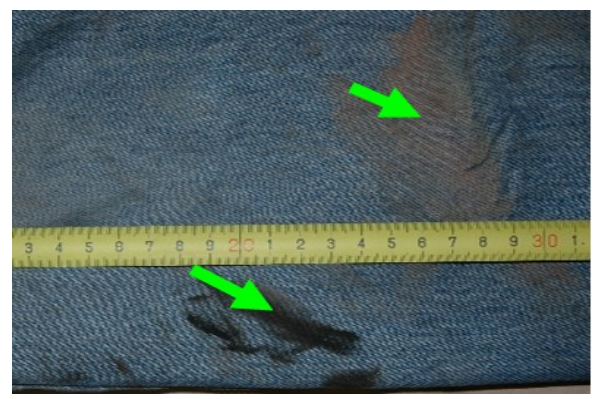

a)

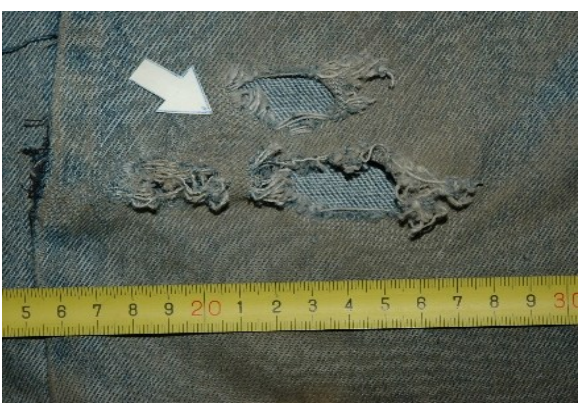

b)

Fig. 1. The victim's trousers after the accident 


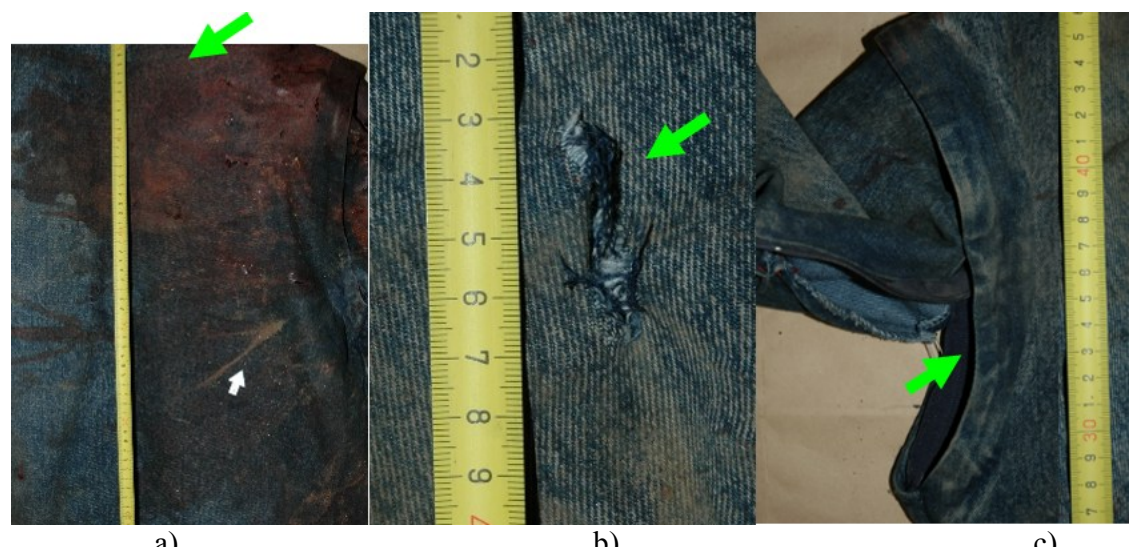

a)

Fig. 2. The victim's jacket after the accident b)

The legal medical examination of the victim's body revealed that the lesional image was dominated by a multitude of injuries - bruises, muscle ruptures, wounds, fractures, mostly placed above the victim's centre of gravity, above the lombar area. These injuries were impossible to individualise with respect to one single of the simple lesional mechanisms. Still, it could be determined that the impacting and dragging mechanisms had been dominant, specifically indicating the impact with large vehicles (such as trucks or buses).

Based on these morphological elements, it was concluded that in this case the lesional mechanism was a complex one (impacting-falling-dragging).

This led to the hypothesis of an impact with a large vehicle, with a high ground clearance. This was proven by identifying a vehicle matching these characteristics only a few streets away from the accident site.

Often the authors of such accidents, after fleeing the accident site, attempt to remove or hide any traces of the accident, in most cases acting on the visible traces, located in easily accessible areas of the vehicle but omitting traces in places that are more difficult to access, such as the parts below the vehicle's axle.

In this case, the examination of the suspect vehicle aimed at searching for traces (biological traces/microtraces, textile traces/microtraces, imprints created by the impact, etc.) left by the victim on the vehicle chassis and the various vehicle parts at the moment of impact.

Following the criminalistic examination of the truck, there was noticed damage at the right headlamp and at the bumper (Figure 3).

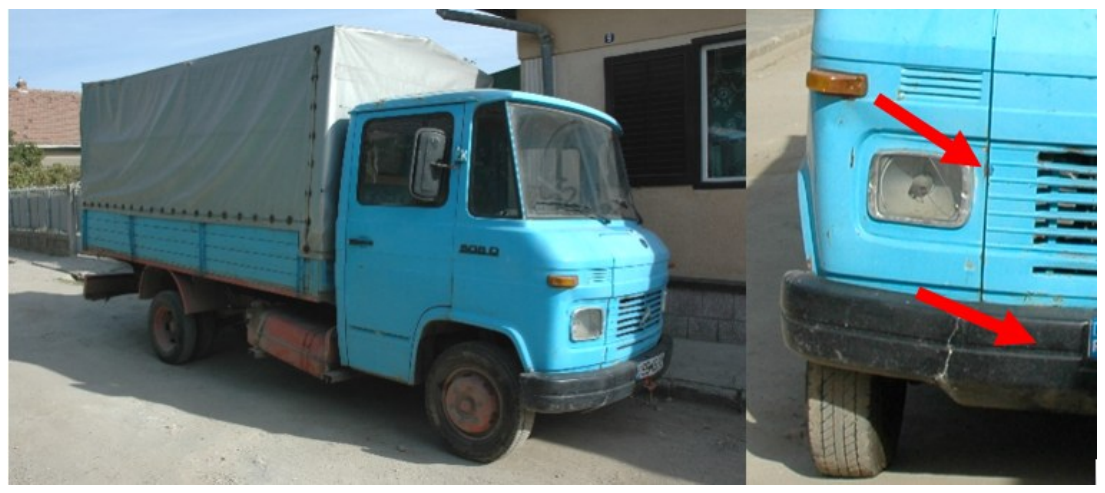

Fig. 3. The truck suspected to have caused the accident 
Under the truck platform there were identified transfer traces of an oily substance and of the dust from subassemblies from the front part: front engine yoke (1), oil pan (2), front axle (3), drive shaft (4), back yoke (5), as well as on the fuel tank (6), as well as traces of deposition of textile fibres and microfibres on the oil pan (2) (Figure 4).

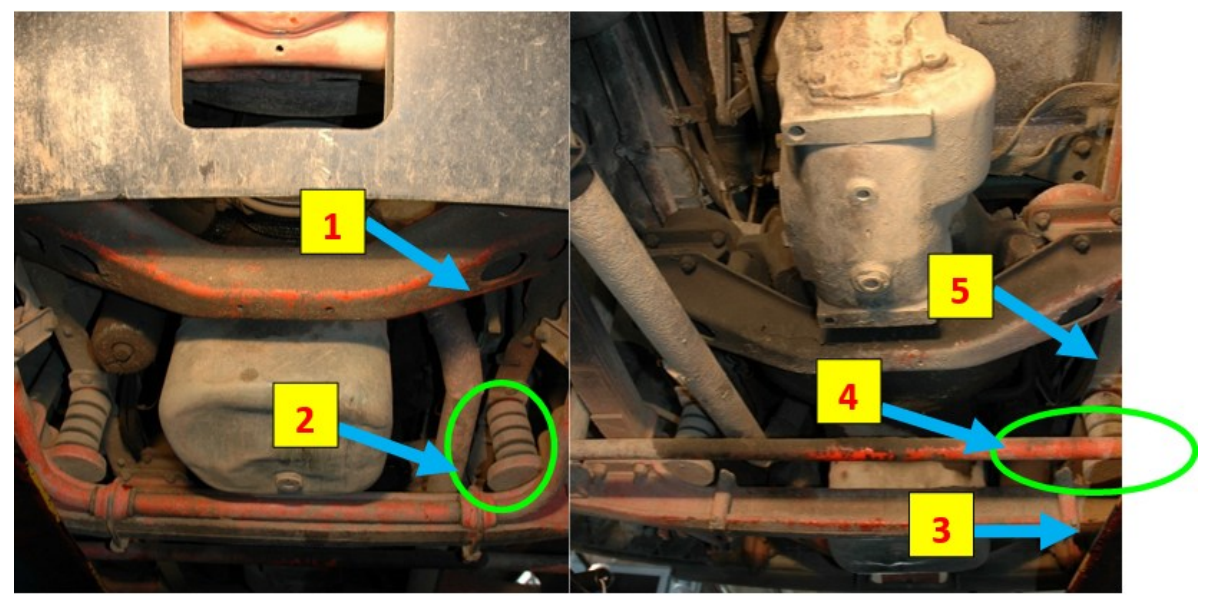

Fig. 4. Photos from under the truck suspected to have caused the accident

The impact traces created by a soil component (possibly created through the contact with textile material/fabric) on the inside-facing part of the fuel tank (Figure $5 \mathrm{a}$ and $\mathrm{b}$ ) and the traces of textile microfibres with a blue colour dominance extracted from the surface of the oil pan (Figure $5 \mathrm{c}$ ), all discovered on the suspected vehicle, as well as the traces of oil and soil on the victim's clothes represented the scientific proof in this case.

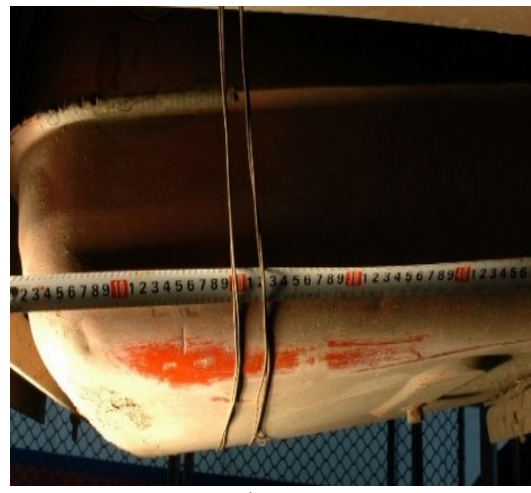

a)

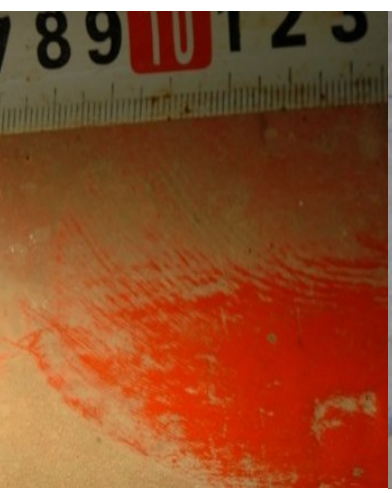

b)

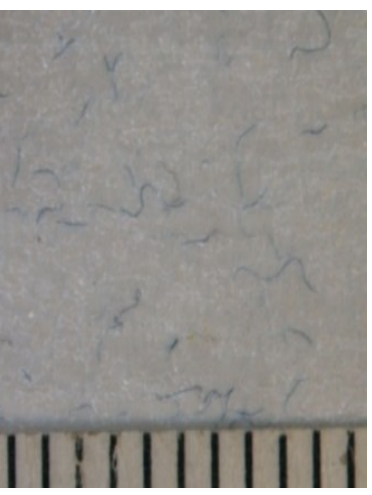

c)

Fig. 5. Elements used as proof for the involvement of the truck in the accident

A subsequent physical-chemical expertise to determine the characteristics and nature of the various materials and substances identified in the previous stages, the crime experts determined two important aspects:

- the generic origin of the blue textile microfibres collected from the oil pan of the truck was the victim's jeans-type trouser material.

- the oil traces transferred onto the victim's trousers (Figure 1 a) could have originated on the suspect truck's subassemblies, showing several similarities.

Furthermore, after a tracking expertise, the forensic experts proved that the traces on the suspect truck's fuel tank were created by the victim's jacket, noticing that between the two 
there were similarities with regard to the general characteristics, having the same linear elements (rows), with similar distancing and alignment (Figure 6).

All these elements corroborated the conclusions reached initially based on the identification of the lesional mechanism, which emphasizes even more the importance of this step in the analysis of road accidents that lead to human victims.

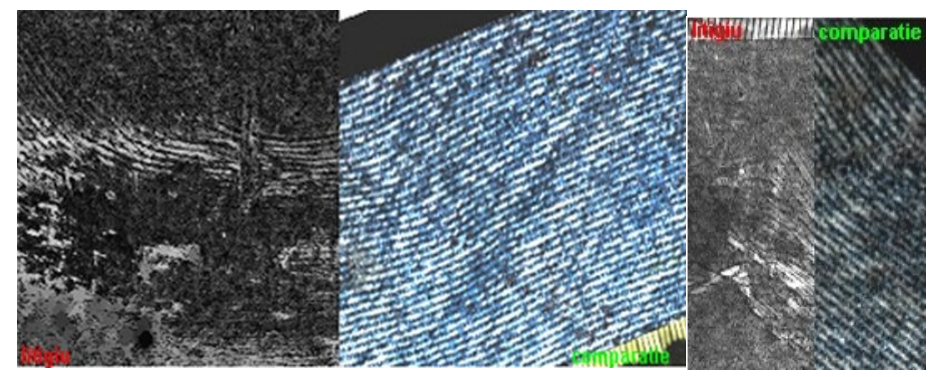

a) b)

Fig. 6. Linear continuity between the jacket's texture and the traces taken from the truck

\subsection{Second case study: impacting-falling-compressing complex mechanism}

The second case analysed by the authors was a road accident produced on the national road DN 7, in the area of the town of Talmaciu, Sibiu county, Romania, on 18.11.2014. The victim was the driver of a truck that got overturned as a consequence of the accident. Also present at the accident site was a badly damaged van (Figures 7-8).

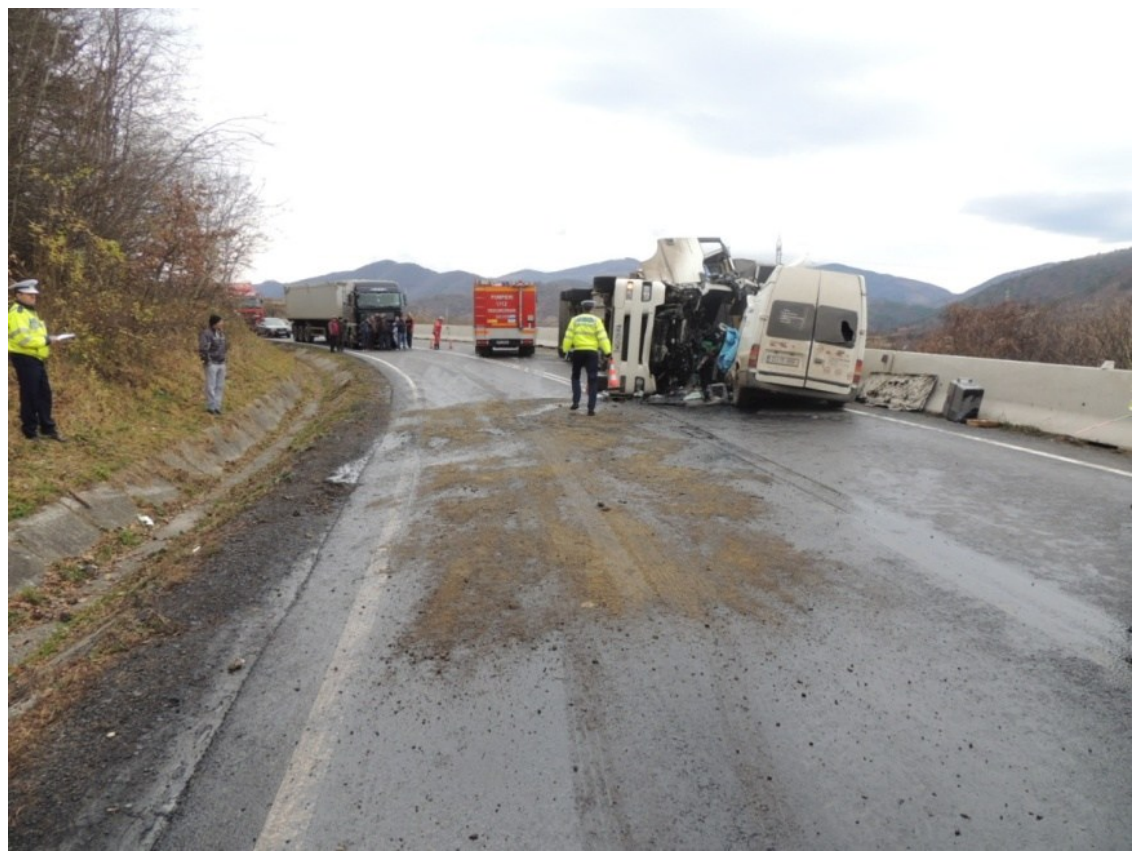

Fig. 7. Site of the second accident 


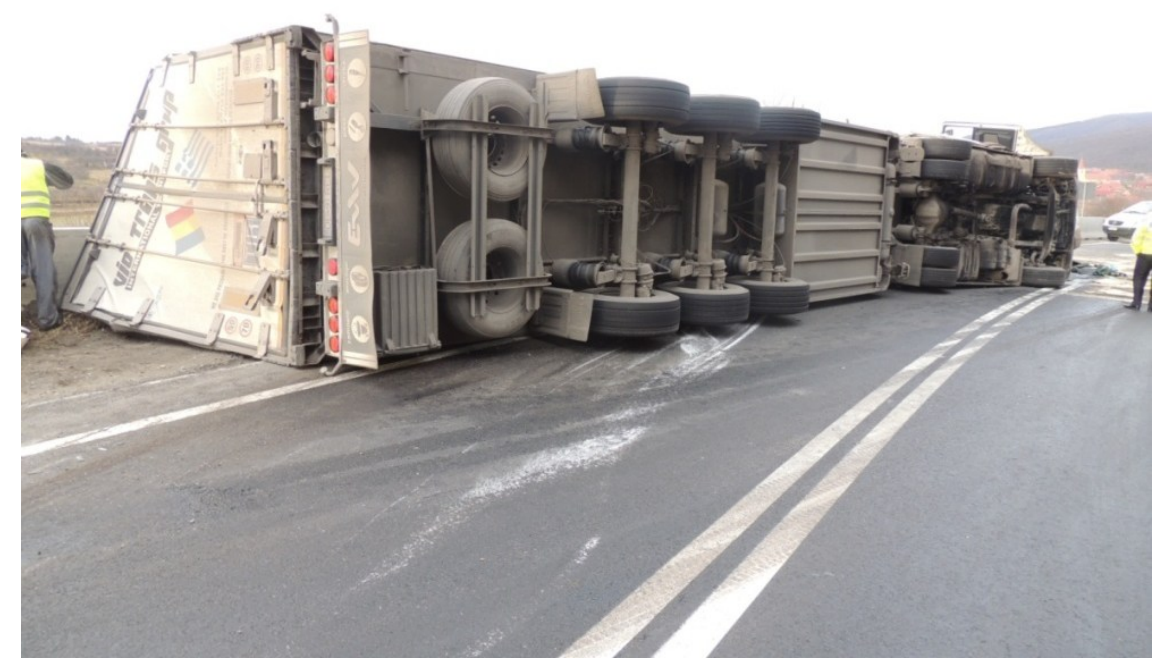

Fig. 8. Wreckage of the truck (rear side)

During the forensic analysis of the truck and van there were noticed multiple and significant damages to the entire chassis of both vehicles, as well as to the elements and systems that directly contribute to safe driving on the road: steering mechanism, braking system, drive system. This made it very difficult to identify the lesional mechanism and to adequately interpret it during the initial phase of the accident investigation.

The legal medical examination of the victim's body indicated that the morphological aspect of the lesions on the victim was a very varied one: bruises, muscle ruptures, severe internal injuries, open wounds, mostly located above the centre of gravity of the victim's body, above the lombar area. While these injuries were impossible to individualise with respect to one single of the simple lesional mechanisms, it could be determined that the impacting and compressing mechanisms had been dominant, specifically indicating the impact with large vehicles (such as trucks or buses).

On the occasion of the forensic examination of the victim's clothing, there were discovered traces of oil and soil, also noticing the presence of material ripping.

It was therefore possible to conclude that in this case, the injuries were produced through the complex impacting-falling-compressing mechanism.

This insight was decisive for the deduction that the incident was caused by the vehicle's speed not being adapted to the road conditions which led to crossing over into the other driving direction and to the violent frontal and side impact between the truck and the van.

\subsection{Third case study: impacting-falling-compressing}

The third analysed case is that of an accident involving two pedestrian victims on a pedestrian road crossing and a passenger bus that happened on 14.10.2014 in the area of the town of Saliste, Sibiu county, Romania.

The legal medical examination of the victims' bodies indicated that the morphological aspect of the lesions on the victim was again a very varied one: bruises, muscle ruptures, open wounds, bone fractures, mostly located above the centre of gravity of the victim's body, above the lombar area. While these injuries were impossible to individualise with respect to one single of the simple lesional mechanisms, there could be separately identified injuries specific for impact and for projecting, respectively and it could be determined that 
these two mechanisms had been dominant, specifically indicating the impact with large vehicles (such as trucks or buses).

The frontal-left side of the bus that seemed to be involved also displayed damage up to a man's height (figure 9).

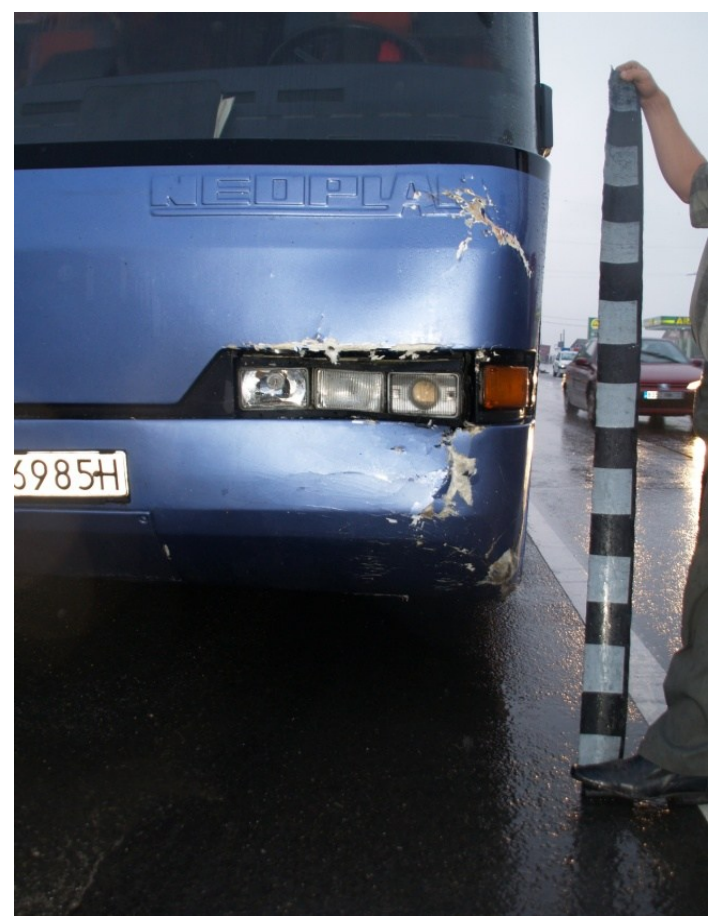

Fig. 9. Damage on the bus involved in the third accident

It was therefore possible to conclude that in this case, the injuries were produced through the complex impacting-falling-projecting-impacting mechanism.

Thus, it could be concluded that the accident was caused by the bus driver hitting the two pedestrians that were legally crossing the street.

\section{Conclusions}

The paper presented the main types of lesional mechanisms occurring in the case of road accidents and showed the importance of correctly identifying these mechanisms for solving forensic aspects of road accidents.

As has been shown in the three case studies, often the identification of individual simple mechanisms can be difficult. Nonetheless, a careful analysis of all injuries on victims' bodies has revealed dominant simple mechanisms, thus allowing the contouring of the complex lesional mechanisms that were generated by the accidents. While the presented cases displayed some common elements, there were also particularities that ultimately helped to precisely identify the circumstances of accidents.

Even though in the cases discussed in this paper, the full identification of all aspects of the accidents and the determining of the guilty parties resulted from a laborious activity unfolded by several specialists from multiple areas, the precise determination of the mechanisms that led to the traumatic injuries and its adequate interpretation in the initial 
stages of the investigation were ultimately the elements leading to the successful solving of the accidents and to serving justice both to the guilty parties and to the victims' families.

\section{References}

1. A. Gligor, V. Petrescu, Matec Web Conf., 184 (2018)

2. M. Dumitru, M. Stanciu, M. Bibu, Lucr. St. Manag. Agr., 15 (2013)

3. M. Bibu, M. Dumitru, Sci. Pap. Manag. Ec. Eng. Agri. Rural Dev., 14 (2014)

4. European Commission, Roadmap to a single European transport area - towards a competitive and resource efficient transport system (European Commission, Brussels, 2011)

5. ETSC, Ranking EU progress on road safety. 14th road safety performance index report (European Transport Safety Council, Brussels, 2020)

6. ERSO, Annual accident report 2018 (European Road Safety Observatory, 2019)

7. K. Goniewicz, M. Goniewicz, W. Pawłowski, D. Lasota, Journal of Education, Health and Sport, 7, 7 (2017)

8. V. Lapadusi, Gh. Popa, D. Voinea, I. Stefan, Criminalistic Investigation of Road Accidents. Contribution of Mass-Media in Preventing Them (in Romanian), (Association of Criminalists of Romania, Bucharest, 2008)

9. L.C. Balan, Adv Soc Sci Edu Hum, 211 (2018)

10. P.L. Munteanu, M. Rosu, V. Panaitescu, A. Punga, Rom. J. Legal Med., 22, 1 (2014) 